\title{
Alternatives Discussed at Indian Science Congress
}

Bhubaneswar, India, January 3-7, 2012

The $99^{\text {th }}$ annual Indian Science Congress was held at Bhubaneswar, the capital of Odisha, India. The Kalinga Institute of Information Technology (KIIT) University, in academic partnership with the National Institute of Science, Education and Research (NISER) hosted the event during January 3-7. The focal theme of the Congress was Science and Technology for Inclusive Innovation - Role of Women. More than 15,000 delegates, including scientists and students, and six laureates participated in the event.

For the first time, a plenary session on alternative methods entitled Animal Alternatives in Teaching and Testing was held at the ISC. Interest in alternatives has been spurred by the success of the Mahatma Gandhi-Doerenkamp Center (MGDC) for Alternatives to Use of Animals in Life Science Education, established at Bharathidasan University, Tiruchirappalli, Tamil Nadu, by the Doerenkamp-Zbinden Foundation and People for Animals, Chennai, the only 3Rs institute in India, in phasing out animal dissections in higher education in a decision of the University Grants Commission (UGC), the regulatory authority of higher education in India, and led to an invitation to the MGDC to organize the session.

The session took place on January 6, 2012 with Dr Rallapalli Ramamurthy as the convener of the session and Dr Mohammad Abdulkader Akbarsha, Director, MGDC, as the chairman. Dr Ramamurthy, also the former Vice-Chancellor, Sree Venkateswara University, Tirupati, India, introduced the theme of the session and stressed the need for inculcating and practicing 3Rs in scientific pursuit. Dr Akbarsha, also the Gandhi-Gruber-Doerenkamp Chair, thanked the ISCA for recognizing the science of $3 \mathrm{Rs}$ as an area of active research and nurture.

"For a long time animal testing has been considered as a gold-standard for any kind of toxicity testing, but the value of animal testing is often overestimated," said Dr Thomas Hartung, Doerenkamp-Zbinden Chair for Evidence-Based Toxicology, Johns Hopkins University, USA. Many experiments in animals have been shown to lead to false-positive or false-negative results. An interesting case is Aspirin, the first chemical entity introduced and used as a drug in 1897. After a variety of tests in animal models it is found to be severely toxic, in fact fatal, to animals. Its $\mathrm{LD}_{50}$ in rats is equivalent to the therapeutic dose in humans! If subjected to regulatory testing as currently prescribed, it would not qualify to be declared as a drug for human consumption at all. Therefore, animal experiments are not $100 \%$ reliable. "A rat is not a $70 \mathrm{~kg}$ man," he said. About 70 million chemicals have been synthesized and about 100,000 of them are found in consumer products, whereas only a negligible number have gone through toxicity evaluation. If all these chemical entities were to be tested, an astronomical number of animals would need to be sacrificed and each test would cost about US\$ 1.5 million. He pointed out that modern toxicological tools and approaches, including in vitro and in silico methods, can largely substitute or complement traditional animal tests. One such approach is mapping the human pathways of toxicity (PoT), which will possibly lead to a better understanding of pathway perturbations and give insights into the mode of action of a drug or toxicant. He is leading a team of scientists towards this pursuit in the NIH-sponsored "Human Toxome" Project.

Dr Marcel Leist, Doerenkamp-Zbinden Chair for in vitro toxicology and biomedicine, University of Konstanz, Germany, elaborated upon validation of alternative methods for toxicity testing. The validation process should ensure reproducibility, relevance, and hypothesis-generating potential of the test method. "The field of cosmetics is a good example for progress in establishment and validation of alternative methods, for example the local lymph node assay, in vitro eye corrosion test and in vitro eye irritation tests have OECD acceptance and have substituted the corresponding animal experiments to a large extent," he said. More than 82 methods have been validated thus, of which 50 are in vitro tests, 10 use isolated organs, and the others are refined in vivo methods in which the test modalities have been improved so as to be more humane to animals. Many of the validated tests are now accepted by the OECD and the European Pharmacopoeia. Referring to the huge gap in developmental neurotoxicity he stated, "The available data regarding the developmental neurotoxicity of industrial chemicals is rather limited. For some compounds developmental neurotoxicity is the most sensitive of all toxicity endpoints evaluated in a broad safety evaluation battery. Thus, although developmental neurotoxicity appears to be an important domain of safety evaluation, test capacity is limited and test costs are extremely high. This puts pressure on the development of faster and cheaper in vitro systems that can predict developmental neurotoxicity, give information comparable to behavioral readouts, and facilitate screening or at least prioritization of relevant drugs and chemicals for further testing."

Dr Albert P. Li, CEO, AP Sciences Inc., Columbia, USA, spoke on the novel toxicity testing system known as Integrated discrete Multiple Organ Co-culture (IdMOC), which he invented and patented. The IdMOC system mimics the interaction between different cell types within an organ or between cells from multiple organs in a plastic dish. He stated, "IdMOC is a valuable experimental system for multiple cell type drug metabolism studies." For example, the cancer chemotherapy drug cyclophosphamide does not directly affect the target organs/tissues but it does so after liver metabolism. On the other 
hand, liver metabolism can be different between species and the toxicity evaluation done in the rat and mouse system may not produce data that could be extrapolated to each other or to humans. The IdMOC system makes use of human cells and so the data can be applied immediately to humans. Numerous studies have shown that IdMOC produces results similar to the human system and is an apt modality for making xenobiotic toxic assessments. "IdMOC allows the evaluation of biological effects of chemicals in an easy, accurate, and controlled manner," he stated. IdMOC is an example of a physiologically relevant "in vitro-in vivo" human model for evaluation of chemical toxicity and has the potential to replace and/or reduce animal use in the drug development process.

Dr Hossein Hosseinkhani, Associate Professor, National Taiwan University of Science and Technology, Taiwan, explained the utility of tissue engineering in the development of in vitro alternative models in toxicity testing. His interdisciplinary research merges biomaterials science, nanotechnology, and biological principles to generate $3 \mathrm{D}$ in vitro living organs called "human-on-chip". In this miniaturized cell culture system, a 3-dimensional hydrogel scaffold is produced on microslides. This can be populated by cells from two or more organs, developed from stem cells, to grow separately as 3-dimensional systems. The test compound is first metabolized by one cell system/organ and the metabolite/secretory product then can interact with the other cell system(s)/organs through microchannels in the hydrogel. This 3D model mimics human organs/tissues and aims to reduce the extent of in vivo animal testing. He envisioned that his group's study will be useful for in vitro diagnostics and drug screening applications.

Dr Shiranee Pereira, Senior Scientist, Central Institute of Brackish-water Aquaculture (CIBA), Indian Council of Agricultural Research (ICAR), Chennai, highlighted the advances in molecular biology, biotechnology, and bioinformatic tools, which, if adopted, can replace and/or reduce animal use in toxicity testing. She emphasized that the scientific prowess of alternative methods is well established as evidenced by the Tox-21 and ToxCast initiatives by US EPA where thousands of chemicals are being tested for their safety using in vitro methods. The AXLR8 and SEN-SI-TIV programs funded by the EU will also help revolutionize toxicity testing. At this juncture she summarized the principles of Ahimsa and urged the scientific community to be sensitive to the philosophy of the Great Mahatma. Mohandas Karamchand Gandhi abhorred animal use in scientific discoveries and as proud Indians we should incorporate the principles of Ahimsa in our scientific endeavors. "The science of alternatives is to know that humane science is better science, giving a three-fold advantage of being precise, predictive, and pain-free," she said.
Dr Balakrishna Murthy, Director, International Institute of Biotechnology and Toxicology (IIBAT), Padappai, India, was of the opinion that if validated in vitro test systems are not available, animal testing remains unavoidable in the context of regulatory requirements. Under such circumstances only the minimum number of animals should be used (reduction alternative), and the experimental procedure should be improved such that the animals are dealt with in a humane manner (refinement alternative). The labs where the tests are conducted should fulfill the internationally acceptable standards, i.e., they should be GLP certified. He outlined that Indian regulators still require old modalities of tests for registering a chemical entity. He cited the example of an agro-chemical entity which has passed all animal testing before being licensed for marketing and use. If the same entity is brought up for regulatory approval by another company it has to undergo all the tests again. "It is time that repetition of such studies is discouraged; risk assessment should be made with already available data." Registration guidelines in India should be modified to reduce animal testing.

Dr Surendra Ghaskadbi, Head, Division of Animal Sciences, Agharkar Research Institute, Pune, stated that when animal use is unavoidable, instead of using animals with higher sentience, such as monkeys, rabbits, rats, mice, and guinea pigs, simple, less sentient organisms should be used for toxicity testing, provided these animals also manifest the same/similar endpoints. He pointed out that many invertebrates like Drosophila, Caenorhabditis elegans, and Daphnia can be employed for screening of toxic substances and can be incorporated as a tiered-testing system for risk assessment. Hydra, a much simpler aquatic organism, is being developed as a model for aquatic toxicity testing in his lab, in collaboration with MGDC.

Dr Krishan K. Sharma, Dean - Postgraduate Studies, Maharshi Dayanand Saraswati University, Ajmer, called for the removal of wild animals from display in museums and from use for identification purposes and systematics studies. Molecular systematics, which uses DNA tests, requires a very small sample without killing the animal, and it can be very reliably used for species identification. Moreover, sound-producing animals can be accurately identified from the uniqueness of their calls. Thus, a new branch of science called sonotaxonomy is emerging. He demonstrated the calls of closely related species of frogs.

Dr Mukkara C. Sathyanarayana, Visiting Faculty, MGDC, was critical of the outdated practice of animal dissection as laboratory exercises. The removal of animals from their habitat for dissection can potentially affect the biodiversity. Dissection is only a tool to understand animal anat- 
omy. With the advances in information and communication technology, anatomy can be learned better by using humane digital alternatives. He also emphasized the concept of "live zoology" wherein students learn about animals in the field by observing rather than dissecting them in the laboratory.

Dr Akbarsha closed the session by stating that "Alternatives is no more a philosophy of ethics; it is no more even the concept of 3Rs; it has come to be science - core science. It has come to be an arena of scientific research applying the latest developments in science and technology towards finding newer alternatives to animal use in teaching, research, testing, and drug development. Thus, 'alternatives' is no more a practice to adopt but a cutting edge science of research to discover and also validate alternative methods."

The General President of ISCA, Dr Geetha Bali, recognized MGDC's efforts in organizing the session and stated, "The deliberations will have an impact on the science policy of the country. This is one of the sessions that will draw a lot of attention from all quarters. We must ensure that the recommendations of the Congress in respect to alternatives be prepared in the best possible way and be submitted to the Government of India."

In light of the recent advances of alternative methods in India, i.e., teachers of more than 500 colleges have been sensitized about digital alternatives to replace dissections and in vitro alternatives to replace in vivo testing; in eight workshops more than 100 young researchers have been trained in cell culture techniques and in vitro toxicology; the University Grants Commission has imposed new strong and stringent guidelines for animal use in education; the Indian National Science Academy (INSA) and the Committee for the Purpose of Control and Supervision of Experiments on Animals (CPCSEA), Govt. of India, have discussed the advantages of in vitro testing; and the ISC has hosted this special plenary session, it was suggested that India might establish a Center for the Validation of Alternative Methods, similar to, e.g., ECVAM and ICCVAM, and/or a Center for Alternatives to Animal Testing, similar to CAAT and CAAT-Europe.

\section{Acknowledgements}

The financial support towards the travel of speakers from abroad by the Doerenkamp-Zbinden Foundation, Switzerland; Advanced Pharmaceutical Sciences Inc, Columbia, USA; and I-CARE, Italy, is heartily acknowledged. The overwhelming support in organizing this special plenary session extended by Dr Geetha Bali and Dr Rallapalli Ramamurthy is also specially acknowledged.

\author{
Mohammad A Akbarsha ${ }^{1}$, \\ Mohammed Zeeshan ${ }^{l}$, \\ and Shiranee Pereira ${ }^{2}$ \\ ${ }^{1}$ Mahatma Gandhi-Doerenkamp \\ Center for Alternatives to Use \\ of Animals in Life Science \\ Education \& Gandhi-Gruber- \\ Doerenkamp Chair, Bharathidasan \\ University, Tiruchirappalli, India \\ ${ }^{2}$ CIBA, Indian Council for \\ Agricultural Research, Chennai, India
}

